\title{
Unusual Abdominal Wall Abscess Post-Laparoscopic Cholecystectomy
}

\author{
Prashanth Annayyanapalya Thimmegowda ${ }^{1}$, Krish Lakshman ${ }^{2}$ \\ ${ }^{1}$ Department of General Surgery, Sagar Hospital, Bengaluru, Karnataka, India \\ ${ }^{2}$ General, Laparoscopic and Gastrointestinal Surgeon, Shanthi Hospital and Research Centre, \\ Bengaluru, Karnataka, India
}

Correspondence to: Dr. Prashanth Annayyanapalya Thimmegowda; email: at.prashanth@gmail.com

Received: 3 Jun 2020; Revised: 17 Aug 2020; Accepted: 08 Sep 2020; Available online: 10 Mar 2021

\section{Summary}

Cholecystectomy is the most commonly performed operation worldwide nowadays. Laparoscopic cholecystectomy (LC) is the gold standard treatment of gallstones. We present a case of an 81-year-old male with a 3-months' history of loss of appetite and weight with no associated symptoms. The patient had undergone an LC for symptomatic cholelithiasis 1 year previously, with an uneventful recovery. The clinical examination was essentially normal. A computed tomography (CT) of the abdomen and pelvis showed thickening of the right perihepatic peritoneum measuring $15 \times 15 \times 3.5 \mathrm{~cm}$, suggestive of chronic granulomatous lesion or atypical mesothelioma. We performed a diagnostic laparoscopy and found the lesion to be an abdominal wall abscess. The abscess cavity was deroofed, the pus was drained and a thorough wash out given. Surprisingly no stones or any foreign body were found in the cavity. Histology of the abscess wall showed non-specific inflammation. We report this case as a post-LC abdominal wall abscess with two peculiar features - (a) no systemic or local symptoms, and (b) no association with spilt gallstones or other foreign bodies like sutures.

Keywords: Parietal wall abscess, laparoscopic cholecystectomy

Ann Afr Surg. 2021; 18(3):185-188

DOI: http://dx.doi.org/10.4314/aas.v18i3.11

Conflicts of Interest: None

Funding: None

(C) 2021 Author. This work is licensed under the Creative Commons Attribution 4.0 International License.

\section{Introduction}

Cholecystectomy is the most commonly performed general surgical operation worldwide (1). Laparoscopic cholecystectomy (LC) is currently the gold standard treatment for gallstones. The most frequent complications are gallbladder perforation, gallstone spillage, and bile duct injuries (2). The incidence of gallbladder perforation and split gallstones were $18.3 \%$ and $7.3 \%$, respectively, in a review by an Australian group which included around 18,000 laparoscopic cholecystectomies. They reported that around one third of split gallstones were left unretrieved (3).

Patients may present with an intraperitoneal or abdominal wall abscess secondary to retained gallstones after an uncomplicated LC at any time and it accounts

for approximately $14 \%$ of the complications from retained gallstones and the majority were along the port sites (4-6). There have been many studies attributing the abdominal wall abscess post-LC to retained gallstones or bile spillage but the occurrence of an abdominal wall abscess without these underlying causes is rare.

We report one such case of an abdominal wall abscess presenting 1 year after LC and describe laparoscopic drainage as our choice of management for the chronic abscess post-LC.

\section{Case report}

An 81-year-old male presented with complaints of loss of weight and appetite for 3 months. There were no 
associated symptoms like fever, nausea, vomiting, or bowel and bladder disturbances. Past medical history was non-contributory other than his only previous surgery. He had undergone an uncomplicated LC for symptomatic cholelithiasis 1 year previously. There was no spillage of bile or gall stones during the surgery. His post-operative course was uneventful. On general physical examination, the patient was afebrile with a soft, non-distended and non-tender abdomen. The port sites were normal. There were no signs of inflammation like fullness, erythema, or tenderness. No palpable lumps were felt anywhere in the abdomen. There was no clinical evidence of any abscess or infection at at the port sites.

Routine bloods were within normal limits. An ultrasound of the abdomen showed multiple ill-defined lesions $2-6 \mathrm{~cm}$ in size in the liver and also a mass of $6 \times$ $2.5 \mathrm{~cm}$ was seen in the right lobe of the liver raising the suspicion of a neoplastic lesion. A computed tomography (CT) of the abdomen and pelvis showed thickening of the right perihepatic peritoneum measuring $15 \times 15 \times 3.5 \mathrm{~cm}$ which was indenting the right lobe hepatic convexity associated with perihepatic fluid collection, suggestive of a chronic granulomatous state or an atypical mesothelioma (Figure 1). There were no space occupying lesions in the liver as was reported in the ultrasound examination. An upper gastrointestinal (GI) endoscopy and colonoscopy showed normal mucosa.

A diagnostic laparoscopy was performed. It showed a bulge in the abdominal wall in the right hypochondrium. There were no granulomatous lesions anywhere intraperitoneally. The bulge was explored and it was found to be an abscess cavity. The cavity was deroofed and around $30 \mathrm{~mL}$ of frank pus was drained. The cavity was washed out. Intraoperative pictures at different stages of the surgery are shown in Figure 2. A Gram stain of the pus showed plenty of pus cells and no organisms. A culture at the end of $48 \mathrm{~h}$ yields no growth of any organism. Histopathology showed fibro-fatty tissue with few muscle bundles.

Dense collections of acute inflammatory cell collection were present in this tissue. The final impression was consistent with an abscess. The patient was on antibiotics for 5 days. The follow-up was uneventful. He remained well with a return of normal appetite in 2 weeks. He remained asymptomatic at the 15-month follow-up. As he was asymptomatic, no further investigations were done.

\section{Discussion}

The gold standard treatment for gallstones is LC due to its known advantages. However, this procedure also brings some risks like gallbladder perforation with bile spillage, gallstone spillage, and bile duct injuries. Patients may present with complications like intra or extra abdominal abscesses due to bile spillage or retained gallstones at any time following surgery.

If we look at the causes for retained gallstones and bile spillage, Nooghabi et al. (7) in a review article noted that an increased incidence was seen when surgery was performed for an acutely inflamed gallbladder, in the elderly, in obese patients, in males, and also in the presence of adhesions. In order to prevent bile spillage, careful dissection and identification of the correct planes should be done during removal of the gallbladder from the liver bed. Decompression can be done in the case of a distended gallbladder. Gallstones and bile spillage can also occur at the time of specimen retrieval and the best way to avoid this is to use an endobag. This prevents port site contamination and retention of gallstones in the port sites. If any spillage occurs at all at the time of dissection or specimen retrieval, the surgeon should ensure removal of all visible stones and use copious saline irrigation to flush the port site (8).

However, in our case we did not find any gallstone in the abscess cavity. The majority of intra or extra abdominal abscesses were caused by spilt gallstones during primary surgery but in our case, there was no bile spillage or retained gallstones. The cause for the abscess in our case remains an enigma. We can only guess that this may be (a) a port site infection tracking obliquely towards the peritoneum or (b) an unrelated abscess in the abdominal wall due to seeding from a bacteremia. 

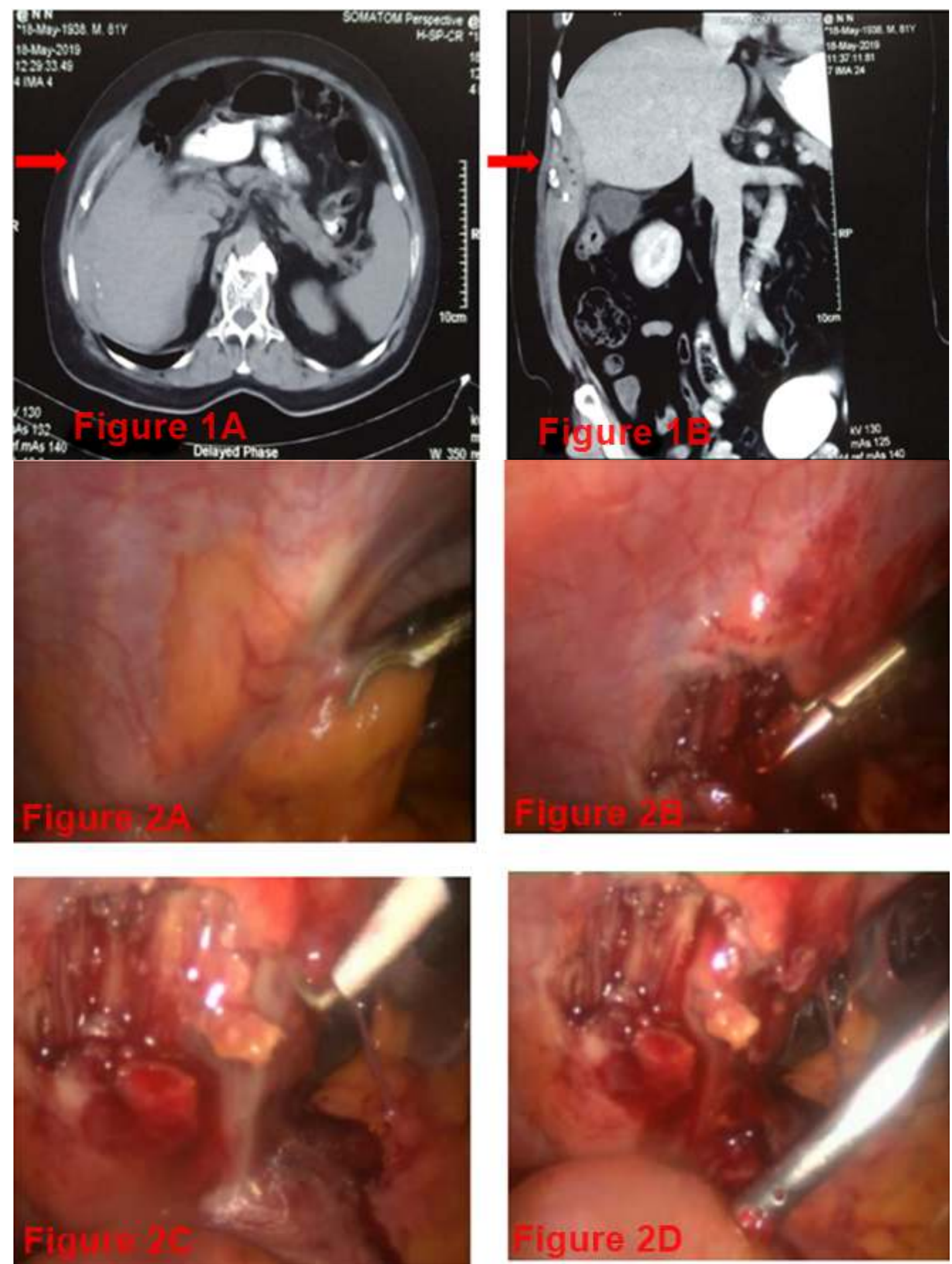

Figure 1. CT of the abdomen and pelvis; Figure 2: Intraoperative pictures at different stages of the surgery 


\section{Conclusion}

Despite the majority of patients remaining asymptomatic, intra or extra abdominal abscesses can occur at any time post-LC. Thorough investigation and timely intervention, including a laparoscopic approach is the key to management.

\section{Informed consent}

This study was conducted in compliance with ethical standards. Informed consent was obtained from the patient.

\section{References}

1. Fingar KR, Stocks C, Weiss AJ, et al. Most frequent operating room procedures performed in US Hospitals, 2003-2012 Statistical Brief \#186. Bethesda, MD: National Centre for Biotechnolofy Information.

2. Mahabaleshwar V, Kaman L, Iqbal J, et al. Monopolar electrocautery versus ultrasonic dissection of the gallbladder from the gallbladder bed in laparoscopic cholecystectomy: a randomized controlled trial. Can J Surg. 2012; 55(5): 307.

3. Woodfield JC, Rodgers M, Windsor JA. Peritoneal gallstones following laparoscopic cholecystectomy. Surg Endosc. 2004; 18(8): 1200-1207.

4. Grass F, Fournier I, Bettschart V. Abdominal wall abscess after cholecystectomy. BMC Res Notes. 2015; 8(1): 334.

5. Christensen AM, Christensen MM. Abdominal wall abscess containing gallstones as a late complication to laparoscopic cholecystectomy performed 17 years earlier. J Surg Case Rep. 2013; 2013(1).

6. Dobradin A, Jugmohan S, Dabul L. Gallstone-related abdominal abscess 8 years after laparoscopic cholecystectomy. J Soc Laparoendoscopic Surgeons. 2013; 17(1): 139-142.

7. Nooghabi AJ, Hassanpour M, Jangjoo A. Consequences of lost gallstones during laparoscopic cholecystectomy: a review article. Surg Laparoscopy Endoscopy Percutaneous Tech. 2016; 26(3): 183-192.

8. Heywood S, Wagstaff B, Tait N. An unusual site of gallstones five years after laparoscopic cholecystectomy. Internat J Surg Case Rep. 2019; 56: 107-109. 